\title{
ON THE CORRESPONDENCE PRINCIPLE FOR THE QUANTIZED ANNULUS
}

\author{
MIROSLAV ENGLIŠ and JAAK PEETRE
}

\section{Introduction.}

A general scheme for quantization has been proposed by Berezin (see e.g. [B1], [B2],...). This scheme involves as main ingredients a complex manifold $\Omega$ equipped with a measure $d \mu$ and a family of Hermitean line bundles $\mathscr{L}_{n}$ depending on a parameter $\hbar \rightarrow 0$ (interpreted as "Planck's constant"). (Actually, nowhere in Berezin's work one finds line bundles; this point of view was introduced by [P0].) Let $A^{2}\left(\Omega, \mu, \mathscr{L}_{\hbar}\right)$ be the Hilbert space of square $\mu$-integrable sections of $\mathscr{L}_{h}$. Then to each linear, say, bounded operator $T$ on $A^{2}\left(\Omega, \mu, \mathscr{L}_{n}\right)$ there corresponds a scalar function $f$, its covariant symbol in the sense of Berezin; the assignment $f \mapsto T_{f}$ is the quantization rule. Furthermore, operator multiplication induces a multiplication of functions (symbols), written $(f, g) \mapsto f *_{n} g$; formally: $T_{f * h g}=T_{f} \circ T_{g}$.

REMARK (on geometric quantization). A related point of view appears in geometric quantization (as developed by Kirillov, Kostant, Lichnerowicz, Souriau and others). A comparison with Berezin's theory is made in Cahen, Gutt and Rawnsley [CGR1]; Section 1 of that paper contains also an excellent introduction to geometric quantization on the whole.

If $\Omega$ is a Kähler manifold, there is a natural choice for $d \mu$ : it is the KählerLiouville (symplectic) measure, while for $\mathscr{L}_{n}$ one can take powers of the canonical bundle (possibly even fractional ones). In this situation there arises the natural question whether one can recover the Kähler or symplectic structure from the multiplication of symbols. We speak of the correspondence principle. In terms of the so-called Berezin transform $B_{h}$ (definition below in a special case) acting on symbols, this involves expanding, for each $f$, the function $B_{h} f$ as a series in powers of $\hbar$. The zeroth term is expected to be just $f$ (i.e. we have then $B_{h} f \rightarrow f$ as $h \rightarrow 0$ ). If the first order term happens to be $\hbar \tilde{\Delta} f$, whee $\tilde{\Delta}$ is the Laplace-Beltrami operator, - a very lucky situation! - one finds that

Received September 30, 1994. 


$$
\lim _{n \rightarrow 0} \frac{1}{i \hbar}\left(f *_{n} g-g *_{n} f\right)=\{f, g\},
$$

where \{\} is the Poisson backet.

Specializing further we let $\Omega \subset \mathrm{C}$ be a planar domain of hyperbolic type and $d s=\frac{|d z|}{\omega(z)}$ the Poincaré metric on $\Omega$. Then we have the Bergman spaces $A_{\alpha}^{2}(\Omega)$ of analytic functions on $\Omega$ square integrable against $\omega(z)^{\alpha} d E(z)$, where $d E$ is the Lebesgue area measure, $\alpha$ and $\hbar$ being related by the formula $\hbar=\frac{1}{\alpha+2}$; thus $\alpha>-1$ and $\hbar>0$. In what follows we shall use as a label instead of $\hbar$ the parameter $\alpha$. It can be shown that $A_{\alpha}^{2}(\Omega)$ are Hilbert spaces admitting a reproducing kernel $K_{\alpha}(x, \bar{y})$, i.e.

$$
f(y)=\int_{\Omega} f(x) \overline{K_{\alpha}(x, \bar{y})} \omega(x)^{\alpha} d E(x) \quad \forall f \in A_{\alpha}^{2}(\Omega) .
$$

The Berezin transform corresponding to $A_{\alpha}^{2}(\Omega)$ is the integral operator on bounded functions on $\Omega$ given by

$$
B_{\alpha} f(y)=\int_{\Omega} f(x) \frac{K_{\alpha}(x, \bar{y}) K_{\alpha}(y, \bar{x})}{K_{\alpha}(y, \bar{y})} \omega(x)^{\alpha} d E(x) .
$$

In this paper we will mostly be interested in the particular case when $\Omega$ is the annulus $\mathrm{A}_{R}=\{z \in \mathrm{C}: 1<|z|<R\}$. It was then shown in [P1] that, for functions $f$ continuous on the closure of $A_{R}$,

$$
B_{\alpha} f \rightarrow f \text { as } \alpha \rightarrow+\infty ;
$$

this is a weak form of the correspondence principle. Our main result (Theorem 5) here will be the following "strong" correspondence principle: if $f$ is a polynomial in $z$ and $\bar{z}$, then

$$
B_{\alpha} f=f+\frac{1}{\alpha} \omega(z)^{2} \Delta f+O\left(\alpha^{-2}\right) \quad \text { as } \alpha \rightarrow+\infty .
$$

Here $\Delta=\frac{\partial^{2}}{\partial z \partial \bar{z}}$ is the usual Euclidean Laplace operator on $C$ and $\tilde{\Delta}=\omega(z)^{2} \Delta$ is the Laplace-Beltrami operator on $\mathrm{A}_{R}$ corresponding to the Poincare metric $d s^{2}$.

An important ingredient in the proof of Theorem 5 will be the fact (Theorem 1) that

$$
\lim \frac{K_{\alpha}(z, \bar{z}) \omega(z)^{\alpha+2}}{\alpha}=\frac{1}{\pi}
$$


as $\alpha$ tends to infinity through the set of even integers. This seems to be closely related to an analogous result of Cahen, Gutt and Rawnsley [CGR2] for compact Kähler manifolds. Note that the quantity $K_{\alpha}(z, \bar{z}) \omega(z)^{\alpha+2}$ has a meaningful analog also in the general case of a Kähler manifold equipped with a line bundle, with which we began this discussion. It plays a rôle also, for instance, in the theory of Hankel forms [JPR].

We also obtain a similar asymptotic estimate for the limiting case $R=+\infty$, i.e. for the punctured disc $A_{0}=\{z \in C: 0<|z|<1\}$ (Theorem 7).

For the annulus itself we find also (Theorem 6) the second term in the asymptotic expansion of the Berezin transform.

At any rate, even from the point of view of Riemann surfaces it is a very special situation that we are considering. Accordingly also the method used is adapted to the situation at hand. It depends on the orthogonal development of functions in the special basis $z^{p}, p=0, \pm 1, \pm 2, \ldots$ and performing quite explicit calculations.

It would be interesting to obtain analogous formulas for general Riemann surfaces. In this direction some results have been obtained by the junior author. In particular, he has been able to obtain a generalization of Theorem 1 [E].

REMARK. As the referee also points out, actually $\alpha+2\left(=\frac{1}{\hbar}\right)$ is the natural parameter, not $\alpha$. However, the passage to $\alpha+2$ does not lead to any major simplification of our formulae. Therefore, in order also to conform with [P1] and other papers, we have decided to work with $\alpha$ after all.

\section{Asymptotics of the reproducing kernels.}

From now on, we take $\Omega$ to be the annulus $A_{R}=\{z \in \mathrm{C}: 1<|z|<R\}$. The Poincaré metric $\frac{|d z|}{\omega}$, corresponding to the metric $\frac{|d z|}{2 \operatorname{Im} z}$ on the upper half-plane, can be found from the formula in [P2]:

$$
\omega(z)=\frac{2 \log R}{\pi}|z| \sin \frac{\pi \log |z|}{\log R} .
$$

The factor $\frac{2 \log R}{\pi}$ was ignored in [P2], but we retain it as it will make our results look more aesthetic; of course, we must keep in mind to modify the formulas from [P2] accordingly.

The functions $z^{p}, p=0, \pm 1, \pm 2, \ldots$, form an orthogonal basis in $A_{\alpha}^{2}\left(\mathrm{~A}_{R}\right)$. For $\alpha$ an integer, their norms are (see [P2], p. 127) 


$$
\left\|z^{p}\right\|_{\alpha}^{2}=2^{\alpha+1} \pi \Lambda^{\alpha+1} \alpha ! \frac{R^{2 p+\alpha+2}+(-1)^{\alpha+1}}{\prod_{k=0}^{\alpha}(\rho+\pi i(\alpha-2 k))},
$$

where we have put

$$
\Lambda=\log R, \quad \rho=(2 p+\alpha+2) \Lambda .
$$

For simplicity, we shall treat only the case when $\alpha$ is even: $\alpha=2 A, A \in Z_{+}$. The last formula then simplifies to

$$
\left\|z^{p}\right\|_{\alpha}^{2}=2^{2 A+1} \pi \Lambda^{2 A+1}(2 A) ! \frac{R^{2(p+A+1)}-1}{\prod_{k=-A}^{+A}(\rho+2 k \pi i)} \stackrel{\text { def }}{=} N_{p}(A) .
$$

Using the parameter $q=p+A+1$, this may be rewritten as

$$
\begin{aligned}
\left\|z^{p}\right\|_{\alpha}^{2} & =2^{2 A+1} \pi \Lambda^{2 A+1}(2 A) ! \frac{R^{2 q}-1}{\prod_{k=-A}^{+A}(2 q \Lambda+2 k \pi i)} \\
& =2^{2 A+1} \pi \Lambda^{2 A+1}(2 A) ! \frac{R^{2 q}-1}{2 \Lambda q \prod_{k=1}^{A}\left(4 \Lambda^{2} q^{2}+4 \pi^{2} k^{2}\right)} \stackrel{\text { def }}{=} M_{q}(A),
\end{aligned}
$$

with the understanding that for $q=0$

$$
\left.\frac{R^{2 q}-1}{q}\right|_{q=0}=2 \log R\left(=\lim _{q \rightarrow 0} \frac{R^{2 q}-1}{q}\right) .
$$

The reproducing kernels are then given by ([P2], p. 129)

$$
K_{A}(x, \bar{y})=\sum_{p \in Z} \frac{(x \bar{y})^{p}}{N_{p}(A)}=(x \bar{y})^{-A-1} \sum_{q \in Z} \frac{(x \bar{y})^{q}}{M_{q}(A)},
$$

where, abusively, we write simply $K_{A}$ instead of $K_{\alpha}$. For $A=\alpha=0$, this sum admits a closed expression in terms of the Weierstrass $\not$-function $([\mathrm{Bg}]$, p. 10):

$$
\begin{aligned}
K_{0}(x, \bar{y}) & =\frac{1}{\pi x \bar{y}}\left[\not 2(\log x \bar{y})+\frac{\eta}{\pi i}-\frac{1}{2 \Lambda}\right]= \\
& \left.=\frac{1}{\pi x \bar{y}}[\not \log x \bar{y})-\frac{\eta^{\prime}}{\Lambda}\right],
\end{aligned}
$$

where $\not$ is the Weierstrass elliptic function with fundamental periods $(2 \pi i,-2 \Lambda)$, and $\eta=\zeta(\pi i), \eta^{\prime}=\zeta(-\Lambda)$ are the half-period increments of the Weierstrass $\zeta$ function with the same period lattice. Here we have used the Legendre relation (see [A], Table V, or [MOS], p. 390)

$$
\eta \cdot(-\Lambda)-\eta^{\prime} \cdot(\pi i)=\frac{\pi i}{2}
$$


Our main goal in this section is the following

THEOREM 1. With the notations as above,

$$
\lim _{A \rightarrow+\infty} \frac{K_{A}(z, \bar{z}) \omega(z)^{2 A+2}}{2 A}=\frac{1}{\pi}
$$

uniformly on $A_{R}$. More precisely, there is an asymptotic estimate

$$
\frac{\pi K_{A}(z, \bar{z}) \omega(z)^{2 A+2}}{2 A+1}=1+O\left(\gamma^{-A}\right) \text { uniformly on } \mathrm{A}_{R},
$$

where

$$
\gamma=\frac{1+\cosh \left(2 \pi^{2} / \Lambda\right)}{2}>1
$$

Proof. From (4) we get

$$
|z|^{2 A+2} K_{A}(z, \bar{z})=\sum_{q \in Z} \frac{|z|^{2 q}}{M_{q}(A)} .
$$

Denote, for brevity, $|z|^{2}=t$. Using (3), we see that

$$
|z|^{2 A+2} K_{A}(z, \bar{z})=k_{A}(\log t),
$$

where

$$
\begin{aligned}
k_{A}(u)= & \sum_{q \in Z} \frac{e^{q u}}{M_{q}(A)}=\frac{1}{(2 \Lambda)^{2 A+1} \pi} \sum_{q \in Z} \sum_{k=1}^{A} \frac{4 \Lambda^{2 q} q^{2}+4 \Lambda q}{R^{2 q}-1} \cdot \frac{2 \Lambda q}{R^{2 q}-1} e^{q u} \\
& =\left(\frac{\pi}{2 \Lambda}\right)^{2 A} \prod_{k=1}^{A} \frac{4 \Lambda^{2} \frac{d^{2}}{d u^{2}}+4 \pi^{2} k^{2}}{\pi^{2} 2 k(2 k-1)} \frac{1}{\pi} \sum_{q \in Z} \frac{q}{R^{2 q}-1} e^{q u} \\
& =\left(\frac{\pi}{2 \Lambda}\right)^{2 A} \prod_{k=1}^{A} \frac{4 \Lambda^{2} \frac{d^{2}}{d u^{2}}+4 \pi^{2} k^{2}}{\pi^{2} 2 k(2 k-1)} \cdot f(u),
\end{aligned}
$$

where

$$
f(u) \equiv k_{0}(u)=\frac{1}{\pi}\left(\not(u)-\frac{\eta^{\prime}}{\Lambda}\right)
$$

owing to (5). Consider the differential operators

$$
D_{k}:=\frac{4 \Lambda^{2} \frac{d^{2}}{d u^{2}}+4 \pi^{2} k^{2}}{\pi^{2} 2 k(2 k-1)}, \quad k \in Z \backslash\{0\},
$$


and introduce the following notation

$$
(g(u))_{A}:=D_{A} D_{A-1} \ldots D_{1} g(u)
$$

for iterated applications of $D_{k}$; here $g$ is an arbitrary analytic function of $u$. Since

$$
\omega(z)^{2 A+2}=\left(\frac{2 \Lambda}{\pi}\right)^{2 A+2} t^{A+1} \sin ^{2 A+2} \frac{\pi \log t}{2 \Lambda},
$$

the limit which we are interested in is simply

$$
\lim _{A \rightarrow+\infty} \frac{\left(\frac{2 \Lambda}{\pi}\right)^{2}(f(u))_{A} \sin ^{2 A+2} \frac{\pi u}{2 \Lambda}}{2 A+1}, \quad u \equiv \log t \in(0,2 \Lambda) .
$$

Of course, the Weierstrass $\not h$-function with periods $(2 \pi i,-2 \Lambda)$ is the same as the $\not h$-function with periods $(2 \Lambda, 2 \pi i)$, and similarly for $\zeta$-functions. Taking the latter as the fundamental period pair and recalling the formula in [A], Table $X$, or in [MOS], p. 389, we see that

$$
\begin{aligned}
\pi f(u) & =\not h)+\frac{\zeta(\Lambda)}{\Lambda}= \\
& =\frac{\pi^{2}}{4 \Lambda^{2}} \operatorname{cosec}^{2} \frac{\pi u}{2 \Lambda}-\frac{\pi^{2}}{\Lambda^{2}} \sum_{m=1}^{\infty} \frac{m}{K^{m}-1}\left(e^{m \pi i u / \Lambda}+e^{-m \pi i u / \Lambda}\right),
\end{aligned}
$$

where $K=e^{2 \pi^{2} / \Lambda}>1$. Expanding $1 /\left(K^{m}-1\right)$ into a power series

$$
\frac{1}{K^{m}-1}=\sum_{c=1}^{\infty} K^{-c m}
$$

changing the order of summation (which is easily justified), and using the formula

$$
\sum_{m=1}^{\infty} m t^{m}=\frac{t}{(1-t)^{2}}
$$

we obtain

$$
\begin{aligned}
\sum_{m=1}^{\infty} \frac{m}{K^{m}-1} e^{m \pi i u / \Lambda} & =\sum_{c=1}^{\infty} \sum_{m=1}^{\infty} m K^{-c m} e^{m \pi i u / \Lambda} \\
& =\sum_{c=1}^{\infty} \frac{e^{\pi i u / \Lambda} K^{-c}}{\left(1-e^{\pi i u / \Lambda} K^{-c}\right)^{2}}
\end{aligned}
$$

But

$\frac{e^{\pi i u / \Lambda} K^{-c}}{\left(1-e^{\pi i u / \Lambda} K^{-c}\right)^{2}}=\left(e^{\pi i u / 2 \Lambda} K^{-c / 2}-\frac{1}{e^{\pi i u / 2 \Lambda} K^{-c / 2}}\right)^{-2}=\left(2 i \sin \left(\frac{\pi u}{2 \Lambda}+\frac{i c \pi^{2}}{\Lambda}\right)\right)^{-2}$ 
so the last sum is equal to

$$
-\frac{1}{4} \sum_{c=1}^{\infty} \operatorname{cosec}^{2}(s+i c a)
$$

where

$$
s=\frac{\pi u}{2 \Lambda}, \quad a=\frac{\pi^{2}}{\Lambda}
$$

Replacing $s$ by $-s$ and substituting both formulas into (9), we get

$$
\pi f(u)=\frac{\pi^{2}}{4 \Lambda^{2}} \sum_{c \in Z} \sin ^{-2}(s+i c a) .
$$

To obtain $(f(u))_{A}$, we use the following wonderful lemma.

LEMMA 1. For any non-zero integer $k$ and complex number $C$,

$$
D_{k}\left(\sin ^{2 k}\left(\frac{\pi u}{2 \Lambda}+C\right)\right)=\sin ^{2 k-2}\left(\frac{\pi u}{2 \Lambda}+C\right) .
$$

Proof of Lemma 1. Compute:

$$
\begin{aligned}
\frac{d^{2}}{d u^{2}} \sin ^{2 k}\left(\frac{\pi u}{2 \Lambda}\right. & +C)=\frac{d}{d u}\left(2 k \sin ^{2 k-1} \cdot \cos \cdot \frac{\pi}{2 \Lambda}\right) \\
& =2 k(2 k-1) \sin ^{2 k-2} \cdot\left(1-\sin ^{2}\right) \cdot \frac{\pi^{2}}{4 \Lambda^{2}}+2 k \sin ^{2 k-1} \cdot(-\sin ) \cdot \frac{\pi^{2}}{4 \Lambda^{2}} \\
& =\frac{\pi^{2}}{4 \Lambda^{2}} 2 k(2 k-1) \sin ^{2 k-2}-\frac{\pi^{2}}{4 \Lambda^{2}} 4 k^{2} \sin ^{2 k}
\end{aligned}
$$

(we have omitted the argument $\frac{\pi u}{2 \Lambda}+C$ ). Thus,

$$
\left(4 \Lambda^{2} \frac{d^{2}}{d u^{2}}+4 \pi^{2} k^{2}\right) \sin ^{2 k}=\pi^{2} 2 k(2 k-1) \sin ^{2 k-2},
$$

and the lemma follows.

Returning to the proof of the theorem, we get

$$
\begin{aligned}
\left(\operatorname{cosec}^{2}(s+i c a)\right)_{A} & =D_{A} D_{A-1} \ldots D_{1}\left(\sin ^{-2}(s+i c a)\right) \\
& =\frac{2 A+1}{2 A-1} \frac{2 A-1}{2 A-3} \ldots \frac{3}{1} \cdot D_{-A} D_{-A+1} \ldots D_{-1}\left(\sin ^{-2}(s+i c a)\right) \\
& =(2 A+1) \sin ^{-2 A-2}(s+i c a) .
\end{aligned}
$$


Here we have used the "wonderful lemma" (Lemma 1) together with a simple observation

$$
D_{k}=\frac{2 k+1}{2 k-1} D_{-k}, \quad k \neq 0
$$

It follows that

$$
(\pi f(u))_{A}=\frac{\pi^{2}}{4 \Lambda^{2}}(2 A+1) \sum_{c \in Z} \sin ^{-2 A-2}(s+i c a),
$$

and

$$
\frac{\left(\frac{2 \Lambda}{\pi}\right)^{2}(\pi f(u))_{A} \sin ^{2 A+2} \frac{\pi u}{2 \Lambda}}{2 A+1}=\sum_{c \in Z}\left(\frac{\sin s}{\sin (s+i c a)}\right)^{2 A+2}
$$

However,

$$
\begin{aligned}
|\sin (s+i c a)|^{2} & =\sin (s+i c a) \sin (s-i c a)= \\
& =\frac{1}{2}(\cos 2 i c a-\cos 2 s)=\frac{1}{2}(\cosh 2 c a-\cos 2 s) .
\end{aligned}
$$

Therefore

$$
\left|\frac{\sin s}{\sin (s+i c a)}\right|^{2}=\frac{1-\cos 2 s}{\cosh 2 c a-\cos 2 s} \leqq \frac{2}{\cosh 2 c a+1} .
$$

Now we can use the following elementary lemma.

LEMMA 2. Let $\left\{a_{j}\right\}_{j=1}^{\infty}$ be a sequence of positive numbers such that $a_{1}>a_{2} \geqq$ $a_{3} \geqq \ldots$, and assume that $\sum_{j=1}^{\infty} a_{j}^{q}$ converges for some $q>0$. Then

$$
\sum_{j=1}^{\infty} a_{j}^{q}=O\left(a_{1}^{q}\right) \quad \text { as } q \rightarrow+\infty
$$

Proof of Lemma 2. We have

$$
\sum_{j=1}^{\infty} a_{j}^{q}=a_{1}^{q} \cdot\left[1+\left(a_{2} / a_{1}\right)^{q} \sum_{j=2}^{\infty}\left(a_{j} / a_{2}\right)^{q}\right]
$$

but, since $0 \leqq a_{j} / a_{2} \leqq 1$ for $j \geqq 3$, the last sum is a decreasing function of $q$, and we even have

$$
\sum_{j=1}^{\infty} a_{j}^{q}=a_{1}^{q} \cdot\left[1+O\left(\left(a_{2} / a_{1}\right)^{q}\right)\right]=a_{1}^{q}(1+o(1)) \quad \text { as } q \rightarrow+\infty
$$


Setting

$$
a_{j}=\frac{2}{\cosh 2 j a+1},
$$

an application of the lemma shows that

$$
\begin{aligned}
\left|\sum_{c=1}^{\infty}\left(\frac{\sin s}{\sin (s+i c a)}\right)^{2 A+2}\right| & \leqq \sum_{c=1}^{\infty}\left|\frac{\sin s}{\sin (s+i c a)}\right|^{2 A+2} \\
& \leqq \sum_{c=1}^{\infty}\left(\frac{2}{\cosh 2 c a+1}\right)^{A+1}=O\left(\gamma^{-A}\right),
\end{aligned}
$$

where

$$
\gamma=1 / a_{1}=\frac{1+\cosh \left(2 \pi^{2} / \Lambda\right)}{2}>1
$$

Of course, the sum over the negative $c$ 's can be treated in the same way; hence,

$$
\frac{\left(\frac{2 \Lambda}{\pi}\right)^{2}(\pi f(u))_{A} \sin ^{2 A+2} \frac{\pi u}{2 \Lambda}}{2 A+1}=1+O\left(\gamma^{-A}\right),
$$

and Theorem 1 follows.

For future reference, we list the following two corollaries, which are direct consequences of Theorem 1.

Corollary 3. $\lim _{A \rightarrow+\infty} \frac{|z|^{2} K_{A}(z, \bar{z})}{K_{A-1}(z, \bar{z})}=\frac{\pi^{2}}{4 \Lambda^{2}} \operatorname{cosec}^{2} \frac{\pi \log |z|}{\Lambda}$, uniformly on $\mathrm{A}_{R}$.

COROLLARY 4. $\lim _{A \rightarrow+\infty}(A+1)\left(\frac{\pi^{2}}{4 \Lambda^{2}} \frac{K_{A-1}(z, \bar{z})}{|z|^{2} K_{A}(z, \bar{z})}-\sin ^{2} \frac{\pi \log |z|}{\Lambda}\right)$

$=-\sin ^{2} \frac{\pi \log |z|}{\Lambda}$, uniformly on $A_{R}$.

Proof. By Theorem 1,

$$
|z|^{2 A+2} K_{A}(z, \bar{z})=\frac{2 A+1}{\pi}\left(\frac{\pi}{2 \Lambda}\right)^{2 A+2} \sin ^{-2 A-2} \frac{\pi u}{2 \Lambda} \cdot\left(1+O\left(\gamma^{-A}\right)\right) .
$$

Hence,

$$
\frac{\pi^{2}}{4 \Lambda^{2}} \frac{K_{A-1}(z, \bar{z})}{|z|^{2} K_{A}(z, \bar{z})}=\frac{\pi^{2}}{4 \Lambda^{2}} \frac{2 A-1}{2 A+1}\left(\frac{\pi}{2 \Lambda}\right)^{-2} \sin ^{2} \frac{\pi u}{2 \Lambda}\left(1+O\left(\gamma^{-A}\right)\right),
$$


so

$$
\frac{\pi^{2}}{4 \Lambda^{2}} \frac{K_{A-1}(z, \bar{z})}{|z|^{2} K_{A}(z, \bar{z})}-\sin ^{2} \frac{\pi u}{2 \Lambda}=-\frac{2}{2 A+1} \sin ^{2} \frac{\pi u}{2 \Lambda}+O\left(\gamma^{-A}\right),
$$

and the assertion follows.

Problem. To extend the results of this section to $\alpha$ non-integer.

\section{The correspondence principle.}

Consider now the effect of the Berezin transform $B_{\alpha}$ on a "binomial" $z^{k} \bar{z}^{l}(k, l \in Z)$. In [P1], it was shown that

$$
\frac{B_{\alpha} z^{k} \bar{z}^{l}}{z^{k} \bar{z}^{l}}=\sum_{p \in \mathcal{Z}} \frac{N_{p+k+l} N_{p}}{N_{p+k} N_{p+l}} \frac{(z \bar{z})^{p}}{N_{p}} / \sum_{p \in \mathcal{Z}} \frac{(z \bar{z})^{p}}{N_{p}}
$$

and proved that this ratio tends to 1 as $\alpha \rightarrow+\infty$ through the integers. We want to determine the first order term (the coefficient at $\alpha^{-1}$ ) in the asymptotic expansion, as $\alpha \rightarrow+\infty$, of (14). In other words, we want to evaluate the limit

$$
\lim _{\alpha \rightarrow+\infty}(\alpha+2)\left(\frac{B_{\alpha} z^{k} \bar{z}^{l}}{z^{k} \bar{z}^{l}}-1\right)=\lim _{\alpha \rightarrow+\infty}(\alpha+2) \sum_{p \in \mathbb{Z}}\left(\frac{N_{p+k+l} N_{p}}{N_{p+k} N_{p+l}}-1\right) \frac{(z \bar{z})^{p}}{N_{p}} / \sum_{p \in Z} \frac{(z \bar{z})^{p}}{N_{p}} .
$$

(The computations below indicate that $\alpha+2$ is a more natural parameter than $\alpha$.) For simplicity, we will again assume that $\alpha=2 A$ is an even integer. From (2) we get

$$
\begin{aligned}
N_{p} & =(2 \Lambda)^{2 A+1} \pi(2 A) ! \frac{R^{2 q}-1}{\prod_{k=-A}^{+A}(2 \Lambda q+2 k \pi i)} \\
& =(2 \Lambda)^{2 A+1} \pi(2 A) ! \frac{R^{2 q}-1}{(2 \pi i)^{2 A+1} \prod_{k=-A}^{+A}\left(\frac{\Lambda q}{\pi i}+k\right)} \\
& =\frac{\Lambda^{2 A+1}(2 A) !}{\pi^{2 A} i(-1)^{A}}\left(R^{2 q}-1\right) \cdot \frac{\Gamma\left(\frac{\Lambda q}{\pi i}-A\right)}{\Gamma\left(\frac{\Lambda q}{\pi i}+A+1\right)}
\end{aligned}
$$

where we have again introduced the parameter $q=p+A+1$. Recalling the functional equation for the $\Gamma$ function, we have 


$$
\begin{aligned}
\Gamma\left(\frac{\Lambda q}{\pi i}-A\right) & =\frac{\pi}{\sin \pi\left(\frac{\Lambda q}{\pi i}-A\right)} \cdot \frac{1}{\Gamma\left(-\frac{\Lambda q}{\pi i}+A+1\right)} \\
& =\frac{2 \pi i(-1)^{A} R^{q}}{R^{2 q}-1} \cdot \frac{1}{\Gamma\left(-\frac{\Lambda q}{\pi i}+A+1\right)}
\end{aligned}
$$

Therefore

$$
N_{p}=2 \pi^{1-2 A} \Lambda^{2 A+1}(2 A) ! R^{q} \cdot\left|\Gamma\left(\frac{\Lambda q}{\pi i}+A+1\right)\right|^{-2}
$$

Similar formulas, of course, hold for $p+k, p+l$ and $p+k+l$ in the place of $p$. Introduce an auxiliary function $F_{k l}(x)$ by

$$
\frac{\Gamma\left(x+\frac{\Lambda k}{\pi i}\right) \Gamma\left(x+\frac{\Lambda l}{\pi i}\right)}{\Gamma(x) \Gamma\left(x+\frac{\Lambda(k+l)}{\pi i}\right)}=F_{k l}(x) .
$$

Then

$$
\frac{N_{p+k+l} N_{p}}{N_{p+k} N_{p+l}}=\left|F_{k l}\left(\frac{\Lambda q}{\pi i}+A+1\right)\right|^{2} .
$$

We want to use the formula (see [MOS], p. 12, or [BE], 1.18. (4))

$$
\frac{\Gamma(x+a)}{\Gamma(x+b)}=x^{a-b}\left[1+\frac{1}{2 x}(a-b)(a+b-1)+O\left(|x|^{-2}\right)\right]
$$

which implies that

$$
\begin{aligned}
& \frac{\Gamma(x+a) \Gamma(x+c)}{\Gamma(x+b) \Gamma(x+d)}=x^{a+c-b-d} \\
& {\left[1+\frac{1}{2 x}\left(a^{2}+c^{2}-b^{2}-d^{2}-a-c+b+d\right)+O\left(|x|^{-2}\right)\right],}
\end{aligned}
$$

both formulas being valid when

$$
|\arg x|<\pi-\delta \quad(\delta>0) .
$$

Since $x=\frac{\Lambda q}{\pi i}+A+1$ satisfies (18), an application of (17) is legitimate, and we get 


$$
F_{k l}\left(\frac{\Lambda q}{\pi i}+A+1\right)=1+\frac{1}{2\left(\frac{\Lambda q}{\pi i}+A+1\right)} \cdot \frac{2 \Lambda^{2} k l}{\pi^{2}}+O\left(\frac{1}{A^{2}+q^{2}}\right),
$$

so the right-hand side of (16) equals

$$
\begin{aligned}
1+2 \operatorname{Re}\left[\frac { \Lambda ^ { 2 } k l } { \pi ^ { 2 } } \left(\frac{\Lambda q}{\pi i}+\right.\right. & \left.A+1)^{-1}\right]+O\left(\frac{1}{A^{2}+q^{2}}\right) \\
& =1+2 \Lambda^{2} k l \frac{A+1}{\Lambda^{2} q^{2}+\pi^{2}(A+1)^{2}}+O\left(\frac{1}{A^{2}+q^{2}}\right) .
\end{aligned}
$$

It follows that

$$
\begin{aligned}
(A+1)\left(\frac{N_{p+k+l} N_{p}}{N_{p+k} N_{p+l}}-1\right) & =2 \Lambda^{2} k l \frac{(A+1)^{2}}{\Lambda^{2} q^{2}+\pi^{2}(A+1)^{2}}+O\left(\frac{A}{A^{2}+q^{2}}\right) \\
& =2 \Lambda^{2} k l \frac{A^{2}}{\Lambda^{2} q^{2}+\pi^{2} A^{2}}+O\left(\frac{1}{A}\right) .
\end{aligned}
$$

The last equality is justified by the fact that $\left(K \equiv \Lambda^{2} q^{2} / \pi^{2}\right)$

$$
\begin{aligned}
\frac{(A+1)^{2}}{K+(A+1)^{2}}-\frac{A^{2}}{K+A^{2}} & =\frac{K \cdot(2 A+1)}{\left(K+A^{2}\right)\left(K+(A+1)^{2}\right)} \\
& \leqq \frac{2 A+1}{K+(A+1)^{2}}=O\left(\frac{1}{A}\right) .
\end{aligned}
$$

Consequently, the limit which we want to evaluate,

$\lim _{A \rightarrow+\infty}(2 A+2) \frac{\sum_{p \in Z}\left(\frac{N_{p+k+l} N_{p}}{N_{p+k} N_{p+l}}-1\right) \frac{t^{p}}{N_{p}}}{\sum_{p \in Z} \frac{t^{p}}{N_{p}}}$

$$
\begin{gathered}
=\lim _{A \rightarrow+\infty} \frac{\sum_{q \in \mathrm{Z}}(2 A+2)\left(\frac{N_{p+k+l} N_{p}}{N_{p+k} N_{p+l}}-1\right) \frac{t^{q}}{M_{q}}}{\sum_{q \in \mathrm{Z}} \frac{t^{q}}{M_{q}}} \\
t \equiv z \bar{z}, q=p+A+1, N_{p} \equiv N_{p}(A) \equiv M_{q}(A) \equiv M_{q},
\end{gathered}
$$

now becomes 


$$
\frac{4 \Lambda^{2} k l}{\pi^{2}} \lim _{A \rightarrow+\infty} \frac{\sum_{p \in Z}\left[\frac{\pi^{2} A^{2}}{\Lambda^{2} q^{2}+\pi^{2} A^{2}}+O\left(\frac{1}{A}\right)\right] \frac{t^{p}}{N_{p}}}{\sum_{p \in Z} \frac{t^{p}}{N_{p}}} .
$$

Of course, since $\frac{t^{p}}{N_{p}}$ is always positive, the term $O(1 / A)$ gives zero contribution to the limit.

It remains to deal with the term $\frac{\pi^{2} A^{2}}{\Lambda^{2} q^{2}+\pi^{2} A^{2}}$. Note that, by (3),

$$
\frac{N_{p}(A)}{N_{p+1}(A-1)}=\frac{M_{q}(A)}{M_{q}(A-1)}=\frac{4 \Lambda^{2} \cdot 2 A(2 A-1)}{4 \Lambda^{2} q^{2}+4 \pi^{2} A^{2}}=\frac{4 \Lambda^{2} A^{2}}{\Lambda^{2} q^{2}+\pi^{2} A^{2}}+O\left(\frac{1}{A}\right) .
$$

Again, the term $O(1 / A)$ will not contribute to the limit, and we have

$$
\begin{aligned}
\lim _{A \rightarrow+\infty} \frac{\sum_{p \in Z} \frac{\pi^{2} A^{2}}{\Lambda^{2} q^{2}+\pi^{2} A^{2}} \frac{t^{p}}{N_{p}}}{\sum_{p \in Z} \frac{t^{p}}{N_{p}}} & =\lim _{A \rightarrow+\infty} \frac{\pi^{2}}{4 \Lambda^{2}} \frac{\sum_{p \in Z} \frac{N_{p}(A)}{N_{p+1}(A-1)} \frac{t^{p}}{N_{p}}}{\sum_{p \in Z} \frac{t^{p}}{N_{p}}} \\
& =\frac{\pi^{2}}{4 \Lambda^{2}} \lim _{A \rightarrow+\infty} \frac{K_{A-1}(z, \bar{z})}{|z|^{2} K_{A}(z, \bar{z})} \quad \text { by }(4) \\
& =\sin ^{2} \frac{\pi \log |z|}{\Lambda} \text { by Corollary 3. }
\end{aligned}
$$

Thus we finally arrive at

$$
\lim _{A \rightarrow+\infty} \frac{\sum_{p \in Z}(2 A+2)\left(\frac{N_{p+k+l} N_{p}}{N_{p+k} N_{p+l}}-1\right) \frac{t^{p}}{N_{p}}}{\sum_{p \in \mathrm{Z}} \frac{t^{p}}{N_{p}}}=\frac{4 \Lambda^{2} k l}{\pi^{2}} \sin ^{2} \frac{\pi \log |z|}{\Lambda},
$$

or, since $\Delta\left(z^{k} \bar{z}^{l}\right)=k l z^{k-1} \bar{z}^{l-1}$,

$$
\lim _{A \rightarrow+\infty}(2 A+2)\left(\frac{B_{2 A}\left(z^{k} \bar{z}^{l}\right)}{z^{k} \bar{z}^{l}}-1\right)=\omega(z)^{2} \frac{\Delta\left(z^{k} \bar{z}^{l}\right)}{z^{k} \bar{z}^{l}}
$$

Summarizing, we have proved

THEOREM 5. Let $f(z)$ be a polynomial in $z$ and $\bar{z}$. Then 


$$
\lim _{\substack{\alpha \rightarrow+\infty, \alpha \text { even integer }}}(\alpha+2)\left(B_{\alpha} f-f\right)=\omega^{2} \Delta f .
$$

In other words,

$$
B_{\alpha} f=f+\frac{1}{\alpha} \tilde{\Delta} f+O\left(\alpha^{-2}\right)
$$

as $\alpha \rightarrow+\infty$ through the even integers, where $\tilde{\Delta}=\omega^{2} \Delta$ is the Laplace-Beltrami operator corresponding to the Poincaré metric on $A_{R}$.

\section{The second coefficient.}

In this section, we will compute the second order coefficient in the asymptotic expansion, with respect to $\alpha+2$, of $(14)($ as $\alpha \rightarrow+\infty)$. The computation is broken into a series of "claims". Let us begin by introducing some more notation: set

$$
\begin{gathered}
B=A+1, \\
C=\frac{\Lambda q}{\pi},
\end{gathered}
$$

where, as before, $A=\alpha / 2$ ( $\alpha$ being an even nonnegative integer) and $q=p+A+1$. We shall also keep our previous notations

$$
s=\frac{\pi u}{2 \Lambda}, \quad a=\frac{\pi^{2}}{\Lambda} .
$$

For a sequence $\left\{a_{q}(A)\right\}_{q \in Z}$ of complex numbers depending on the parameter $A$, define

$$
\mathscr{L}\left(a_{q}(A)\right):=\lim _{A \rightarrow+\infty} \frac{\sum_{p \in Z} a_{q}(A) \frac{t^{p}}{N_{p}}}{\sum_{p \in Z} \frac{t^{p}}{N_{p}}}=\lim _{A \rightarrow+\infty} \frac{\sum_{q \in Z} a_{q}(A) \frac{t^{q}}{M_{q}}}{\sum_{q \in Z} \frac{t^{q}}{M_{q}}} .
$$

Thus, for instance, (24) can be rewritten in short as

$$
\mathscr{L}\left(\frac{A^{2}}{A^{2}+C^{2}}\right)=\sin ^{2} \frac{\pi u}{2 \Lambda} .
$$

The symbol $\mathscr{L}$ is clearly linear, satisfies $\mathscr{L}\left(a_{q}(A)\right)=1$ if $a_{q}(A)=1$ for all $q$ and $A$, and, as we have already observed, the positiveness of all the $t^{q} / M_{q}$ implies that

$$
\mathscr{L}\left(a_{q}(A)\right)=0 \text { if } a_{q}(A)=O(1 / A) \text { uniformly in } q .
$$


Using these properties of $\mathscr{L}$, the second order coefficient we want to evaluate can be expressed as

$$
\begin{aligned}
\lim _{B \rightarrow+\infty}(2 B)^{2}\left(\frac{B_{A} z^{k} \bar{z}^{l}}{z^{k} \bar{z}^{l}}\right. & \left.-1-\frac{4 \Lambda^{2} k l}{2 B \pi^{2}} \sin ^{2} \frac{\pi u}{2 \Lambda}\right) \\
& =\mathscr{L}\left(4 B^{2}\left[\frac{N_{p+k+l} N_{p}}{N_{p+k} N_{p+l}}-1-\frac{2 \Lambda^{2} k l}{B \pi^{2}} \sin ^{2} s\right]\right) .
\end{aligned}
$$

We now need an amplification of the formula (17):

CLAIM 1. In any sector $|\arg x| \leqq \pi-\delta, \delta>0$, the following asymptotic expansion holds:

$$
F_{k l}(x)=\frac{\Gamma\left(x+\frac{\Lambda k}{\pi i}\right) \Gamma\left(x+\frac{\Lambda l}{\pi i}\right)}{\Gamma(x) \Gamma\left(x+\frac{\Lambda(k+l)}{\pi i}\right)}=1+\frac{d}{x}+\frac{e+f i}{x^{2}}+O\left(x^{-3}\right)
$$

where $d, e$ and $f$ are real numbers given by

$$
d=\frac{\Lambda^{2} k l}{\pi^{2}}, \quad e=\frac{1}{2} d(d+1), \quad f=\frac{\Lambda^{3} k l(k+l)}{2 \pi^{3}} .
$$

Proof. We shall employ the asymptotic formula of Barnes [BE, 1.18.(12)]: $\log \Gamma(z+\alpha)=\left(z+\alpha-\frac{1}{2}\right) \log z-z+\log \sqrt{2 \pi}+\sum_{j=1}^{2} \frac{(-1)^{j+1}}{j(j+1) z^{j}} B_{j+1}(\alpha)+O\left(z^{-3}\right)$, which is valid in any sector of the above type; here $B_{j}(\alpha)$ are the Bernoulli polynomials. Taking, successively, $\alpha=\frac{\Lambda k}{\pi i}, \frac{\Lambda l}{\pi i}, 0$ and $\frac{\Lambda(k+l)}{\pi i}$ and adding up the resulting expansions with appropriate signs, the first three terms cancel out, and we get

$$
\log F_{k l}(z)=\frac{R}{2 z}-\frac{S}{6 z^{2}}+O\left(z^{-3}\right)
$$

where

$$
\begin{gathered}
R=B_{2}(\Lambda k / \pi i)+B_{2}(\Lambda l / \pi i)-B_{2}(0)-B_{2}(\Lambda(k+l) / \pi i)=\frac{2 \Lambda^{2} k l}{\pi^{2}}=2 d, \\
S=B_{3}(\Lambda k / \pi i)+B_{3}(\Lambda l / \pi i)-B_{3}(0)-B_{3}(\Lambda(k+l) / \pi i)=\frac{3 \Lambda^{3} k l(k+l)}{i \pi^{3}}-3 d .
\end{gathered}
$$

Exponentiating, we obtain the desired expansion. 
Claim 2. With d, e and $f$ defined by (28) above, we have (29)

$$
\frac{N_{p+k+l} N_{p}}{N_{p+k} N_{p+l}}=1+\frac{2 d B}{B^{2}+C^{2}}+\frac{d^{2}}{B^{2}+C^{2}}+\frac{2 e\left(B^{2}-C^{2}\right)-4 B C f}{\left(B^{2}+C^{2}\right)^{2}}+O\left(B^{-3}\right) .
$$

ProOF. In view of (16),

$$
\frac{N_{p+k+l} N_{p}}{N_{p+k} N_{p+l}}=\left|F_{k l}\left(\frac{\Lambda q}{\pi i}+A+1\right)\right|^{2}=\left|F_{k l}(B-i C)\right|^{2} .
$$

Plugging (27) into this formula gives (29).

CLAim 3. $\mathscr{L}\left(\frac{B^{2}}{B^{2}+C^{2}}\right)=\sin ^{2} s$.

Proof. In view of the estimate (21), this follows from (25).

Claim 4. $\mathscr{L}\left(\frac{B^{4}}{\left(B^{2}+C^{2}\right)^{2}}\right)=\sin ^{4} s$.

Proof. By (3),

$$
\frac{M_{q}(A)}{M_{q}(A-2)}=\left(\frac{2 \Lambda}{\pi}\right)^{4} \cdot \frac{A(A-1 / 2)(A-1)(A-3 / 2)}{\left(A^{2}+C^{2}\right)\left((A-1)^{2}+C^{2}\right)} .
$$

Now by (21),

$$
\frac{A(A-1)}{A^{2}+C^{2}}=\frac{A^{2}}{A^{2}+C^{2}}+O(1 / A)=\frac{B^{2}}{B^{2}+C^{2}}+O(1 / B),
$$

and similarly for $A-1$ in the place of $A$; thus,

$$
\left(\frac{\pi}{2 \Lambda}\right)^{4} \frac{M_{q}(A)}{M_{q}(A-2)}=\frac{B^{4}}{\left(B^{2}+C^{2}\right)^{2}}+O(1 / B) .
$$

It follows that

$$
\begin{aligned}
\mathscr{L}\left(\frac{B^{4}}{\left(B^{2}+C^{2}\right)^{2}}\right) & =\left(\frac{\pi}{2 \Lambda}\right)^{4} \mathscr{L}\left(\frac{M_{q}(A)}{M_{q}(A-2)}\right) \\
& =\left(\frac{\pi}{2 \Lambda}\right)^{4} \lim _{A \rightarrow+\infty} \frac{K_{A-2}(z, \bar{z})}{|z|^{4} K_{A}(z, \bar{z})} \\
& =\sin ^{4} \frac{\pi u}{2 \Lambda} \text { by Corollary } 3 .
\end{aligned}
$$


Claim 5. $\mathscr{L}\left(\frac{B^{2} C^{2}}{\left(B^{2}+C^{2}\right)^{2}}\right)=\sin ^{2} s \cos ^{2} s$.

Proof. Since $B^{2} C^{2}=B^{2}\left(B^{2}+C^{2}\right)-B^{4}$, this follows at once from Claims 3 and 4.

Corollary. $\mathscr{L}\left(\frac{\left(B^{2}-C^{2}\right) B^{2}}{\left(B^{2}+C^{2}\right)^{2}}\right)=\sin ^{4} s-\sin ^{2} s \cos ^{2} s$.

CLAIM 6. The following analogue of Theorem 1 is valid for the derivative of $k_{A}$ :

$\sum_{g \in \mathrm{Z}} \frac{q t^{q}}{M_{q}(A)}=-(2 A+1)(2 A+2) \frac{1}{\pi}\left(\frac{\pi}{2 \Lambda}\right)^{2 A+3} \operatorname{cosec}^{2 A+2} \frac{\pi u}{2 \Lambda}\left(\operatorname{cotg} \frac{\pi u}{2 \Lambda}+o(1)\right)$.

PRoof. Let us recapitulate some facts from the proof of Theorem 1: with the usual notations $t=|z|^{2}$ and $u=\log t$, we have seen that, by (4),

$$
t^{B} K_{A}(z, \bar{z})=\sum_{q \in Z} \frac{t^{q}}{M_{q}}=k_{A}(u)
$$

where, by (6),

$$
\pi k_{A}(u)=\left(\frac{\pi}{2 \Lambda}\right)^{2 A}(\pi f(u))_{A}
$$

and $(\pi f(u))_{A}$ is given by (12). Thus we see that

$$
\begin{gathered}
t^{B} K_{A}(z, \bar{z})=\sum_{q \in Z} \frac{t^{q}}{M_{q}}=\frac{1}{\pi}\left(\frac{\pi}{2 \Lambda}\right)^{2 A+2}(2 A+1) \sum_{c \in Z} \sin ^{-2 A-2}(s+i c a), \\
t=|z|^{2}, \quad u=\log t, \quad s=\pi u / 2 \Lambda .
\end{gathered}
$$

Differentiation with respect to $u$ gives

$$
\sum_{q \in \mathrm{Z}} \frac{q t^{q}}{M_{q}}=-\frac{1}{\pi}\left(\frac{\pi}{2 \Lambda}\right)^{2 A+3}(2 A+1)(2 A+2) \sum_{c \in Z} \frac{\cos (s+i c a)}{\sin ^{2 A+3}(s+i c a)} .
$$

By a computation similar to (13), $\left|\frac{\cos (s+i c a)}{\sin (s+i c a)}\right|^{2}=\frac{\cosh 2 c a+\cos 2 s}{\cosh 2 c a-\cos 2 s} \leqq \frac{\cosh 2 c a+1}{\cosh 2 c a-1} \leqq \frac{\cosh 2 a+1}{\cosh 2 a-1} \equiv \beta^{2}$ (say), for any $c \in Z, c \neq 0$. Employing Lemma 2, we conclude that $\left|\sin ^{2 A+2} s \sum_{c \neq 0} \frac{\cos (s+i c a)}{\sin ^{2 A+3}(s+i c a)}\right| \leqq \beta \sum_{c \neq 0}\left|\frac{\sin s}{\sin (s+i c a)}\right|^{2 A+2}=2 \beta O\left(\gamma^{-A}\right)=O\left(\gamma^{-A}\right)$ as $A \rightarrow+\infty$, where $\gamma>1$ is the constant from Theorem 1. Hence, 


$$
\sin ^{2 A+2} s \sum_{c \in Z} \frac{\cos (s+i c a)}{\sin ^{2 A+3}(s+i c a)}=\operatorname{cotg} s+O\left(\gamma^{-A}\right),
$$

and (32) follows.

Claim 7. $\mathscr{L}\left(C / B^{2}\right)=0$.

Proof. $\mathscr{L}\left(q / B^{2}\right)=\lim _{A \rightarrow+\infty} B^{-2}\left(\sum_{q \in Z} q t^{q} / M_{q}\right) /\left(\sum_{q \in Z} t^{q} / M_{q}\right) ; \quad$ by $\quad$ Theorem 1 and (32), this equals

$$
\begin{aligned}
\lim _{A \rightarrow+\infty} \frac{1}{B^{2}} \frac{-(2 A+1)(2 A+2)(\pi / 2 \Lambda)^{2 A+3} \operatorname{cosec}^{2 A+2} s(\operatorname{cotg} s+o(1))}{(2 A+1)(\pi / 2 \Lambda)^{2 A+2} \operatorname{cosec}^{2 A+2} s(1+o(1))}= \\
=\lim _{B \rightarrow+\infty} \frac{-2 A-2}{B^{2}} \cdot \frac{\pi}{2 \Lambda}(\operatorname{cotg} s+o(1))=0 .
\end{aligned}
$$

Claim 8. $\mathscr{L}\left(\frac{B^{3} C}{\left(B^{2}+C^{2}\right)^{2}}\right)=-\sin ^{3} s \cos s$.

Proof. From (31) we see that

$$
\frac{B^{3} C}{\left(B^{2}+C^{2}\right)^{2}}=\left(\frac{\pi}{2 \Lambda}\right)^{4} \frac{M_{q}(A)}{M_{q}(A-2)} \cdot \frac{C}{B}+O\left(\frac{C}{B^{2}}\right) .
$$

In view of the previous claim, Theorem 1, and (32), we therefore have

$$
\begin{aligned}
& \mathscr{L}\left(\frac{B^{3} C}{\left(B^{1}+C^{2}\right)^{2}}\right)=\left(\frac{\pi}{2 \Lambda}\right)^{4} \mathscr{L}\left(\frac{C}{B} \frac{M_{q}(A)}{M_{q}(A-2)}\right) \\
& =\left(\frac{\pi}{2 \Lambda}\right)^{4} \lim _{B \rightarrow+\infty} \frac{1}{B} \cdot \frac{\Lambda}{\pi} \cdot \frac{\sum_{q \in Z} q t^{q} / M_{q}(A-2)}{\sum_{q \in Z} t^{q} / M_{q}(A)} \\
& =\frac{\Lambda}{\pi}\left(\frac{\pi}{2 \Lambda}\right)^{4} \lim _{B \rightarrow+\infty} \frac{1}{B} \cdot \frac{-(2 A-3)(2 A-2)(\pi / 2 \Lambda)^{2 A-1} \sin ^{-2 A+2} s(\operatorname{cotg} s+o(1))}{(2 A+1)(\pi / 2 \Lambda)^{2 A+2} \sin ^{-2 A-2} s(1+o(1))} \\
& =\frac{\Lambda}{\pi}\left(\frac{\pi}{2 \Lambda}\right)^{4} \lim _{A \rightarrow+\infty} \frac{-(2 A-3)(2 A-2)}{(A+1)(2 A+1)}\left(\frac{\pi}{2 \Lambda}\right)^{-3} \sin ^{4} s(\operatorname{cotg} s+o(1)) \\
& =-\cos s \sin ^{3} s .
\end{aligned}
$$$$
\text { Claim 9. } \mathscr{L}\left(B\left[\frac{\pi^{2}}{4 \Lambda^{2}} \frac{M_{q}(A)}{M_{q}(A-1)}-\sin ^{2} s\right]\right)=-\sin ^{2} s \text {. }
$$

Proof. This quantity equals to

$$
\lim _{B \rightarrow+\infty} B\left(\frac{\pi^{2}}{4 \Lambda^{2}} \frac{K_{A-1}(z, \bar{z})}{|z|^{2} K_{A}(z, \bar{z})}-\sin ^{2} \frac{\pi u}{2 \Lambda}\right),
$$

which in turn is equal to $-\sin ^{2} s$, by Corollary 4 . 
CLAIM 10. $\mathscr{L}\left(B^{2}\left[\frac{B}{B^{2}+C^{2}}-\frac{\sin ^{2} s}{B}\right]\right)=-\frac{1}{2} \sin ^{2} s+2 \sin ^{2} s \cos ^{2} s$.

Proof. By virtue of (23),

$$
\frac{\pi^{2}}{4 \Lambda^{2}} \frac{M_{q}(A)}{M_{q}(A-1)}=\frac{A(A-1 / 2)}{A^{2}+C^{2}}=\frac{A^{2}}{A^{2}+C^{2}}-\frac{1}{2} \frac{A}{A^{2}+C^{2}} .
$$

In view of the estimate (21),

$$
\frac{A^{2}}{A^{2}+C^{2}}-\frac{B^{2}}{B^{2}+C^{2}}=-\frac{C^{2}(2 A+1)}{\left(C^{2}+A^{2}\right)\left(C^{2}+B^{2}\right)}=-\frac{2 B C^{2}}{\left(C^{2}+B^{2}\right)^{2}}+O\left(\frac{1}{B^{2}}\right) \text {. }
$$

A similar estimate shows that

$$
\left|\frac{A}{A^{2}+C^{2}}-\frac{B}{B^{2}+C^{2}}\right|=O\left(\frac{1}{B^{2}}\right) .
$$

Hence,

$$
\frac{\pi^{2}}{4 \Lambda^{2}} \frac{M_{q}(A)}{M_{q}(A-1)}=\frac{B^{2}}{B^{2}+C^{2}}-\frac{2 B C^{2}}{\left(B^{2}+C^{2}\right)^{2}}-\frac{1}{2} \frac{B}{B^{2}+C^{2}}+O\left(B^{-2}\right)
$$

or

$$
\frac{B^{2}}{B^{2}+C^{2}}=\frac{\pi^{2}}{4 \Lambda^{2}} \frac{M_{q}(A)}{M_{q}(A-1)}+\frac{1}{2} \frac{B}{B^{2}+C^{2}}+\frac{2 B C^{2}}{\left(B^{2}+C^{2}\right)^{2}}+O\left(B^{-2}\right) .
$$

Thus,

$$
\begin{aligned}
B^{2}\left[\frac{B}{B^{2}+C^{2}}-\frac{\sin ^{2} s}{B}\right] & =B\left[\frac{\pi^{2}}{4 A^{2}} \frac{M_{q}(A)}{M_{q}(A-1)}-\sin ^{2} s\right] \\
& +\frac{1}{2} \frac{B^{2}}{B^{2}+C^{2}}+\frac{2 B^{2} C^{2}}{\left(B^{2}+C^{2}\right)^{2}}+O(1 / B)
\end{aligned}
$$

and the claims 9,3 and 5 give

$$
\begin{aligned}
\mathscr{L}\left(B^{2}\left[\frac{B}{B^{2}+C^{2}}-\frac{\sin ^{2} s}{B}\right]\right) & =-\sin ^{2} s+\frac{1}{2} \sin ^{2} s+2 \sin ^{2} s \cos ^{2} s \\
& =-\frac{1}{2} \sin ^{2} s+2 \sin ^{2} s \cos ^{2} s
\end{aligned}
$$

as asserted.

Putting all the pieces together, we get from (26) and (29) 


$$
\begin{aligned}
\lim _{A \rightarrow+\infty} & (2 A+2)^{2}\left(\frac{B_{A} z^{k} \bar{z}^{l}}{z^{k} \bar{z}^{l}}-1-\frac{4 \Lambda^{2} k l}{\pi^{2}(2 A+2)} \sin ^{2} s\right)= \\
= & 8 d\left(2 \sin ^{2} s \cos ^{2} s-\frac{1}{2} \sin ^{2} s\right)+4 d^{2} \sin ^{2} s \\
& +8 e\left(\sin ^{4} s-\sin ^{2} s \cos ^{2} s\right)+16 f \cos s \sin ^{3} s
\end{aligned}
$$

where $d, e$ and $f$ are the numbers given by (28). Using the relation $e=d(d+1) / 2$ and a little elementary trigonometry, the right-hand side can be simplified to

$$
8 d \sin ^{2} s+8 d(d-1) \sin ^{4} s+16 f \cos s \sin ^{3} s .
$$

Let us compare this with the action of $\left(\omega^{2} \Delta\right)^{2}$ on $z^{k} \bar{z}^{l}$. We have

$$
\omega^{2} \Delta z^{k} \bar{z}^{l}=\frac{4 \Lambda^{2}}{\pi^{2}}|z|^{2} \sin ^{2} \frac{\pi \log z \bar{z}}{2 \Lambda} \cdot k l z^{k-1} \bar{z}^{l-1}=4 d \sin ^{2} s \cdot z^{k} \bar{z}^{l} .
$$

Consequently,

$$
\begin{aligned}
& \Delta\left(\omega^{2} \Delta z^{k} \bar{z}^{l}\right)=4 d \cdot \frac{\partial}{\partial z}\left[z^{k} \cdot l \bar{z}^{l-1} \sin ^{2} s+z^{k} \bar{z}^{l-1} \cdot 2 \sin s \cos s \cdot \frac{\pi}{2 \Lambda}\right]= \\
& \quad=4 d\left[k l z^{k-1} \bar{z}^{l-1} \sin ^{2} s+z^{k-1} \cdot l \bar{z}^{l-1} \cdot 2 \sin s \cos s \cdot \frac{\pi}{2 \Lambda}+\right. \\
& \left.\quad+k z^{k-1} \bar{z}^{l-1} \cdot 2 \sin s \cos s \cdot \frac{\pi}{2 \Lambda}+z^{k-1} \bar{z}^{l-1} \cdot \frac{\pi}{2 \Lambda} \cdot 2\left(\cos ^{2} s-\sin ^{2} s\right) \cdot \frac{\pi}{2 \Lambda}\right]
\end{aligned}
$$

and

$$
=\frac{4 \Lambda^{2}}{\pi^{2}} \sin ^{2} s \cdot 4 d\left[k l \sin ^{2} s+(k+l) \frac{\pi}{\Lambda} \sin s \cos s+\frac{\pi^{2}}{2 \Lambda^{2}}\left(\cos ^{2} s-\sin ^{2} s\right)\right] z^{k} \bar{z}^{l} .
$$

Hence,

$$
\begin{aligned}
\frac{\left(\omega^{2} \Delta\right)^{2} z^{k} \bar{z}^{l}}{z^{k} \bar{z}^{l}} & =16 d^{2} \sin ^{4} s+\frac{16 \Lambda(k+l)}{\pi} d \cdot \sin ^{3} s \cos s+8 d\left(\sin ^{2} s \cos ^{2} s-\sin ^{4} s\right) \\
& =16 d(d-1) \sin ^{4} s+8 d \sin ^{2} s+32 f \sin ^{3} s \cos s
\end{aligned}
$$

and, owing to (34),

$$
\frac{1}{2} \frac{\left(\omega^{2} \Delta\right)^{2} z^{k} \bar{z}^{l}}{z^{k} \bar{z}^{l}}+\frac{\omega^{2} \Delta z^{k} \bar{z}^{l}}{z^{k} \bar{z}^{l}}=8 d(d-1) \sin ^{4} s+8 d \sin ^{2} s+16 f \sin ^{3} s \cos s
$$

which is the same expression as (33)! Thus, we have finally proved that 
$\lim _{A \rightarrow+\infty}(2 A+2)^{2}\left(\frac{B_{A} z^{k} \bar{z}^{l}}{z^{k} \bar{z}^{l}}-1-\frac{4 \Lambda^{2} k l}{(2 A+2) \pi^{2}} \sin ^{2} \frac{\pi u}{2 \Lambda}\right)=\frac{\left[\frac{1}{2}\left(\omega^{2} \Delta\right)^{2}+\left(\omega^{2} \Delta\right)\right] z^{k} \bar{z}^{l}}{z^{k} \bar{z}^{l}}$

and may summarize our results in the following theorem:

THEOREM 6. Let $f$ be a polynomial in $z$ and $\bar{z}$. Then the asymptotic expansion

$$
B_{\alpha} f=f+\frac{1}{\alpha+2} \tilde{\Delta} f+\frac{1}{(\alpha+2)^{2}}\left(\frac{\tilde{\Delta}^{2}}{2}+\tilde{\Delta}\right) f+O\left(\alpha^{-3}\right)
$$

holds as $\alpha \rightarrow+\infty$ in the set of even integers, where $\tilde{\Delta}=\omega(z)^{2} \Delta$ is the LaplaceBeltrami operator corresponding to the Poincare metric on $A_{R}$.

REMARK. It is clear that the above procedure could be applied to obtain other higher order coefficients as well: slight modifications in the proofs of Claims 4 and 8 show that

$$
\mathscr{L}\left(\frac{B^{k} C^{2 n-k}}{\left(B^{2}+C^{2}\right)^{n}}\right)=(-1)^{k} \sin ^{k} s \cos ^{2 n-k} s,
$$

and the remaining terms, of the type that appears in Claim 10, can be handled by Theorem 1 and (32). Thus, departing from Barnes's formula for $\log \Gamma(z+\alpha)$, it is in principle possible to obtain the whole asymptotic expansion of $B_{\alpha} f$. The calculations involved, however, become increasingly difficult, and so we prefer not to pursue this direction any further.

\section{The punctured disc.}

We conclude by investigating the limiting case $R=+\infty$, or, equivalently, the case when $\Omega$ is the punctured disc

$$
A_{0}=\{z \in C: 0<|z|<1\} .
$$

We begin by computing the Poincaré metric. Introduce the uniformizing parameter

$$
\zeta=-i \log z, \quad \text { or } z=e^{i \xi} \quad(\operatorname{Im} \xi>0)
$$

Then

$$
d \zeta=-i \frac{d z}{z}, \quad \operatorname{Im} \zeta=-\log |z|
$$

and so 


$$
\frac{|d \zeta|}{2 \operatorname{Im} \zeta}=\frac{|d z|}{-2|z| \log |z|}
$$

Thus we are led to take

$$
\omega(z)=-2|z| \log |z| \quad\left(z \in A_{0}\right)
$$

and consider the weighted Bergman spaces $A_{\alpha}^{2}\left(\mathrm{~A}_{0}\right), \alpha \geqq 0$, of analytic functions on $A_{0}$ square integrable with respect to $\omega(z)^{\alpha} d E(z), d E$ being the Lebesgue area measure. Let us compute the $A_{\alpha}^{2}$ norms of the functions $z^{n}, n=0, \pm 1, \pm 2, \ldots$ Using the polar coordinates, we have

$$
\begin{aligned}
\left\|z^{n}\right\|_{A_{\alpha}^{2}}^{2}=\int_{A_{0}}|z|^{2 n} \omega(z)^{\alpha} d E(z)=2 \pi \int_{0}^{1} 2^{\alpha} r^{2 n+\alpha} \log ^{\alpha} \frac{1}{r} \cdot r d r= \\
=2^{\alpha+1} \pi \int_{0}^{+\infty} e^{-(2 n+\alpha+2) t} t^{\alpha} d t \quad\left(t=\log \frac{1}{r}, d r=-e^{-t} d t\right) .
\end{aligned}
$$

For $\alpha \geqq 0$, this is finite if and only if

$$
2 n+\alpha+2>0 \text {. }
$$

Substituting $u=(2 n+\alpha+2) t$, we find

$$
\left\|z^{n}\right\|_{\alpha}^{2}=\frac{2^{\alpha+1} \pi}{(2 n+\alpha+2)^{\alpha+1}} \int_{0}^{+\infty} e^{-u} u^{\alpha} d u=\frac{2^{\alpha+1} \pi \cdot \alpha !}{(2 n+\alpha+2)^{\alpha+1}} \equiv N_{p}(A) .
$$

Looking at Taylor expansions reveals that the functions $\left\{z^{n}\right\}_{n>-(\alpha+2) / 2}$ form an orthogonal basis in $A_{\alpha}^{2}\left(\mathrm{~A}_{0}\right)$. It follows that the reproducing kernels are given by

$$
K_{a}(x, \bar{y})=\frac{1}{\pi} \sum_{n>-\frac{\alpha+2}{2}} \frac{(2 n+\alpha+2)^{\alpha+1}}{2^{\alpha+1} \alpha !}(x \bar{y})^{n}, \quad x, y \in \mathrm{A}_{0} .
$$

For simplicity, we will henceforth consider only the case when $\alpha$ is an even integer: $\alpha=2 A, A \in Z_{+}$. Switching to the parameter $q=n+A+1$, we see that the basis is simply $\left\{z^{q-A-1}\right\}_{q=1}^{\infty}$, while the reproducing kernels are given by

$$
\begin{aligned}
K_{A}(x, \bar{y}) & =\frac{1}{(2 A) ! \pi} \sum_{n=-A}^{\infty}(n+A+1)^{\alpha+1}(x \bar{y})^{n} \\
& =\frac{1}{(2 A) ! \pi} \sum_{q=1}^{\infty}(x \bar{y})^{q-A-1} q^{2 A+1},
\end{aligned}
$$

where, abusively, we write $K_{A}$ instead of $K_{\alpha}$. Thus, again, we see that $(x \bar{y})^{A+1} K_{A}(x, \bar{y})$ is an analytic function of $\log x \bar{y}$ :

$$
(x \bar{y})^{A+1} K_{A}(x, \bar{y})=f_{A}(\log x \bar{y})
$$


where

$$
f_{A}(u)=\frac{1}{(2 A) ! \pi} \sum_{q=1}^{\infty} q^{2 A+1} e^{q u}=\prod_{k=1}^{A}\left(\frac{1}{2 k(2 k-1)} \frac{d^{2}}{d u^{2}}\right) \frac{e^{u}}{\pi\left(1-e^{u}\right)^{2}} .
$$

It follows that

$$
\begin{aligned}
K_{A}(z, \bar{z}) \omega(z)^{2 A+2} & =|\log t|^{2 A+2} \cdot f_{A}(\log t) \\
& =\frac{v^{2 A+2}}{(2 A) ! \pi} \sum_{q=1}^{\infty} q^{2 A+1} e^{-q v}
\end{aligned}
$$

where we have put

$$
t=z \bar{z} \in(0,1), \quad v=-\log t \in(0,+\infty) .
$$

Now we can establish the following analog of Theorem 1.

THEOREM 7. For each $z \in \mathrm{A}_{0}$,

$$
\lim _{A \rightarrow+\infty} \frac{\omega(z)^{2 A+2} K_{A}(z, \bar{z})}{2 A+1}=\frac{1}{\pi}
$$

as $\boldsymbol{A}$ tends to infinity through the integers.

Proof. According to (36),

$$
\frac{\omega(z)^{2 A+2} K_{A}(z, \bar{z})}{2 A+1}=\frac{v^{2 A+2}}{(2 A+1) ! \pi} \sum_{q=1}^{\infty} q^{2 A+1} e^{-q v}=\frac{v^{2 A+2}}{(2 A+1) ! \pi} e^{-v} \Phi\left(e^{-v},-2 A-1,1\right),
$$

where

$$
\Phi(z, s, w)=\sum_{n=0}^{\infty}(w+n)^{-s} z^{n}, \quad|z|<1, w \neq 0,-1,-2, \ldots,
$$

is the Lerch transcendental function. According to Lerch's inversion formula (see [BE], 1.11.(6)),

$$
\begin{aligned}
\Phi(z, s, w)= & z^{-w} \Gamma(1-s) \sum_{n=-\infty}^{+\infty}(-\log z+2 n \pi i)^{s-1} e^{2 n \pi i w}, \\
& 0<w \leqq 1, \operatorname{Re} s<0,|\arg (-\log z+2 n \pi i)| \leqq \pi,
\end{aligned}
$$

so in our case

$$
\Phi\left(e^{-v},-2 A-1,1\right)=e^{v}(2 A+1) ! \sum_{n=-\infty}^{+\infty}(v+2 n \pi i)^{-2 A-2} .
$$

Therefore 


$$
\begin{aligned}
\frac{\omega(z)^{2 A+2} K_{A}(z, \bar{z})}{2 A+1} & =\frac{v^{2 A+2}}{\pi} \sum_{n=-\infty}^{+\infty}(v+2 n \pi i)^{-2 A-2} \\
& =\frac{1}{\pi}+\frac{1}{\pi} \sum_{n \neq 0}\left(\frac{v}{v+2 n \pi i}\right)^{2 A+2}
\end{aligned}
$$

But, as $v \in(0,+\infty)$, Lemma 2 can be applied to

$$
a_{n}=\left|\frac{v}{v+2 n \pi i}\right|^{2}=\frac{v^{2}}{v^{2}+4 \pi^{2} n^{2}}<1 \text { for } n \neq 0,
$$

and it follows that the last sum tends to zero as $A \rightarrow+\infty$. This completes the proof.

Observe that, in this case, the convergence is not uniform on $A_{0}$, but only on subsets of $A_{0}$ that are bounded away from the origin.

\section{REFERENCES}

[A] N. I. Akhiezer, Elements of the Theory of Elliptic Functions, Izd. Nauka, Moscow 1970.

[BE] H. Bateman, A. Erdélyi, Higher Transcendental Functions I, McGraw-Hill, 1953.

[B1] F. A. Berezin, General concept of quantization, Comm. Math. Phys. 40 (1975), 153-174.

[B2] F. A. Berezin, Quantization, Math. USSR - Izv. 8 (1974), 1109-1163.

[Bg] S. Bergman, The kernel function and conformal mapping, Math. Surveys 5 (1950).

[E] M. Engliš, Asymptotics of reproducing kernels on a plane domain, Proc. Amer. Math. Soc. 123 (1995), 3157-3160.

[CGR1] M. Cahen, S. Gutt, J. Rawnsley, Quantization on Kähler manifolds I: Geometric interpretation of Berezin's quantization, J. Geom. Phys. 7 (1990), 45-62.

[CGR2] M. Cahen, S. Gutt, J. Rawnsley, Quantization on Kähler manifolds II, Trans. Amer. Math. Soc. 337 (1993), 73-98.

[JPR] S. Janson, J. Peetre, R. Rochberg, Hankel forms and the Fock space, Rev. Mat. Iberoamericana 3 (1987), 61-138.

[MOS] W. Magnus, F. Oberhettinger, R. Soni, Formulas and Theorems for the Special Functions of Mathematical Physics, Springer-Verlag, 1966.

[PO] J. Peetre, The Berezin transform and Ha-plitz operators, J. Operator Theory 24 (1990), 165-168.

[P1] J. Peetre, Correspondence principle for quantized annulus, Romanowski polynomials, Morse potential, J. Funct. Anal. 117 (1993), 377-400.

[P2] J. Peetre, Hankel forms on multiply connected domains I. The case of connectivity two, Complex Variables Theory Appl. 10 (1988), 123-139.

[PZ] J. Peetre, G. Zhang, Projective structures on an annulus and Hankel forms, Glasgow Math. J. 33 (1991), 247-266.

MATHEMATICAL INSTITUTE

ACADEMY OF SCIENCES

ZITNA 25

11567 PRAGUE 1

CZECH REPUBLIC

email: englis@,csearn.bitnet
MATEMATISKA INSTITUTIONEN

LUNDS UNIVERSITET

BOX 118

S-22100 LUND

SWEDEN

email: jaak@maths.lth.se 